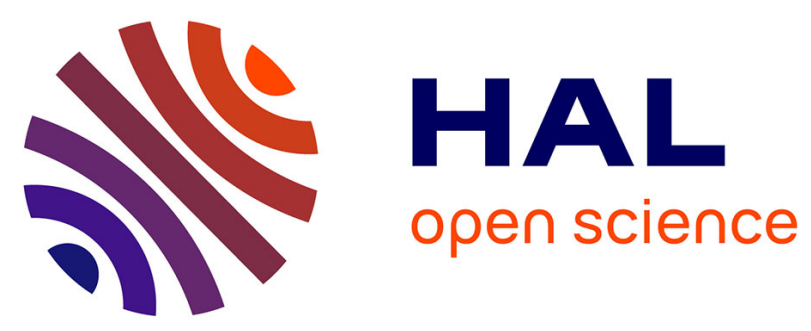

\title{
Comment on "An analysis of VLF electric field spectra measured in Titan's atmosphere by the Huygens probe" by J. A. Morente et al.
}

Réjean Grard, Stéphanie Berthelin, Christian Béghin, Michel Hamelin, Jean-Jacques Berthelier, Jose J. Lopez-Moreno, Fernando Simões

\section{To cite this version:}

Réjean Grard, Stéphanie Berthelin, Christian Béghin, Michel Hamelin, Jean-Jacques Berthelier, et al.. Comment on "An analysis of VLF electric field spectra measured in Titan's atmosphere by the Huygens probe" by J. A. Morente et al.. Journal of Geophysical Research. Planets, 2011, 116, pp.E05005. 10.1029/2009JE003555 . hal-00581649

\section{HAL Id: hal-00581649 \\ https://hal.science/hal-00581649}

Submitted on 12 Mar 2015

HAL is a multi-disciplinary open access archive for the deposit and dissemination of scientific research documents, whether they are published or not. The documents may come from teaching and research institutions in France or abroad, or from public or private research centers.
L'archive ouverte pluridisciplinaire HAL, est destinée au dépôt et à la diffusion de documents scientifiques de niveau recherche, publiés ou non, émanant des établissements d'enseignement et de recherche français ou étrangers, des laboratoires publics ou privés. 


\title{
Comment on "An analysis of VLF electric field spectra measured in Titan's atmosphere by the Huygens probe" by J. A. Morente et al.
}

\author{
Réjean Grard, ${ }^{1,2}$ Stéphanie Berthelin, ${ }^{3}$ Christian Béghin, ${ }^{3}$ Michel Hamelin, ${ }^{4}$ \\ Jean-Jacques Berthelier, ${ }^{4}$ Jose J. López-Moreno, ${ }^{5}$ and Fernando Simões ${ }^{6}$ \\ Received 8 December 2009; revised 9 February 2011; accepted 16 March 2011; published 18 May 2011.
}

Citation: Grard, R., S. Berthelin, C. Béghin, M. Hamelin, J.-J. Berthelier, J. J. López-Moreno, and F. Simões (2011), Comment on "An analysis of VLF electric field spectra measured in Titan's atmosphere by the Huygens probe" by J. A. Morente et al., J. Geophys. Res., 116, E05005, doi:10.1029/2009JE003555.

\section{Introduction}

[1] Morente et al. [2009b] have recently revisited the VLF electric field measurements made with the Permittivity, Wave and Altimetry (PWA) instrument during the descent of the Huygens Probe through the atmosphere of Titan. They assert that they have identified several harmonics of the transverse resonance mode of the surface-ionosphere cavity, which would prove the existence of an electrical activity in the atmosphere of the largest satellite of Saturn. We refute this finding on the basis that it results from an artifact due to an improper analysis of the data set.

[2] The investigators of the Permittivity, Wave and Altimetry (PWA) experiment on the Huygens Probe have reported the extremely low frequency (ELF) and very low frequency (VLF) electric signals recorded during the descent through the atmosphere of Titan [Grard et al., 2006; Béghin et al., 2007; Simões et al., 2007; Béghin et al., 2009]. The PWA data are archived in the Planetary Science Archive (PSA) of ESA, and an extensive description of the instrument is at the disposal of the scientific community [Grard et al., 1995; Fulchignoni et al., 2002; Falkner, 2004]. Morente and his coworkers have revisited this data set and reported the results of their investigations in two papers. In a first paper, they claim that they have detected in the ELF range $(0-100 \mathrm{~Hz})$ several harmonics of a global resonance allegedly generated by lightning activity in the spherical cavity guide formed by the surface of Titan and the inner boundary of the ionosphere, a phenomenon similar to the Schumann resonance observed at Earth [Morente et al., 2008]. In the second paper dedicated to the VLF electric signal recorded by $\mathrm{PWA}$, in the range $0-10 \mathrm{kHz}$, they argue that they can

\footnotetext{
${ }^{1}$ Oegstgeest, Netherlands.

${ }^{2}$ Formerly at RSSD, ESA-ESTEC, European Space Agency, Noordwijk, Netherlands.

${ }^{3}$ LPC2E-CNRS-Université d'Orléans, Orléans, France.

${ }^{4}$ LATMOS-IPSL-Université de Versailles St Quentin, Saint-Maur, France.

${ }^{5}$ Instituto de Astrofisíca de Andalucía IAA-CSIC, Granada, Spain.

${ }^{6}$ NASA Goddard Space Flight Center, Greenbelt, Maryland, USA.

Copyright 2011 by the American Geophysical Union. 0148-0227/11/2009JE003555
}

also bring out the transverse resonance and its harmonics, a more local phenomenon that develops around the excitation source and whose frequency is controlled by the separation between Titan's surface and the inner ionospheric boundary [Morente et al., 2009b].

[3] The PWA investigators have analyzed the narrowband ELF signal at about $36 \mathrm{~Hz}$ effectively observed during the entire descent [Grard et al., 2006; Béghin et al., 2007; Simões et al., 2007] and are still scrutinizing its significance [Béghin et al., 2009]. They have not endorsed, however, the alternative approach of Morente et al. [2008] that discloses additional ELF narrow-band signals and numerous related harmonics. Hamelin et al. [2009] and Béghin et al. [2009] have questioned the work of Morente and his coworkers and demonstrated unambiguously that their findings are mere artifacts entirely due to a faulty procedure. Morente et al. [2009a] refuted this critical analysis of their ELF paper in a rebuttal. The present comment draws a parallelism between the ELF and VLF papers and demonstrates that the VLF signal carries no evidence whatever of any transverse resonance. The analyses of the ELF and VLF signals by Morente and his coworkers are flawed, and their conclusions are shown here to be invalid. A more comprehensive and thorough assessment of the numerical approaches proposed by Morente et al. [2008, 2009b] is given by Berthelin et al. [2009].

\section{A Faulty Procedure}

[4] In a few words, the steps followed by Morente et al. [2008, 2009b] are the following:

[5] 1. The raw ELF, or VLF, energy spectra are extracted from the PSA. The modulus of each spectral component has been obtained by averaging on board the outputs of several direct Fourier transforms (DFT) applied to successive measuring sequences of the signal waveforms. No information on the phases is therefore available. The raw data are neither calibrated nor decompressed.

[6] 2. An inverse DFT is applied to the raw data, assuming that the unknown phases are equal to zero. This operation necessarily yields a temporal signal with a maximum at time zero, since all components are in phase at that time. The magnitude of this peak is the integrated value of 
all raw spectral lines and has nothing to do with any early time impulsive signal.

[7] 3. The initial peak of the reconstructed waveform is blanked out, thus producing a late time signal that should bring up the claimed residual fluctuations in the remainder of the temporal signal.

[8] 4. Zeroes are added to the late time signal, thus artificially lengthening the duration of the time series and consequently increasing the number of frequency lines at the next step.

[9] 5. A direct DFT, followed by another ultimate operation in the case of the VLF signal only (section 3), is applied to the augmented late time signal. These processes yield frequency distributions that exhibit spectral features not visible in the raw data.

[10] The first three steps, though debatable, might possibly help emphasize spectral components in a qualitative way, if they exist and if their amplitudes are larger than the digitization step of the initial spectrum. Even if the raw spectrum contains no significant signatures, as during the interplanetary cruise, the inverse DFT followed by the truncation of the reconstituted pseudosignal will induce new spectral features that are more characteristic of the temporal window than of the original waveform. Adding zeroes to the time series at step 4 does not contribute any information at all; it merely smoothes out the spectrum and provides an interpolation that is not justified, from a mathematical point of view, between adjacent frequency lines. This operation improves, only artificially, the apparent frequency resolution of the subsequent DFT at step 5. As discussed in section 3, as presented in Morente et al.'s Figure 10d the absolute value of the real part of the DFT as a VLF power spectrum [Morente et al., 2009b] is totally unjustified from a physical point of view. Taking the study cases of Morente et al. [2008, 2009b] as examples, we describe in sections 3 and 4 the mechanism that can generate artificial resonances and harmonics out of noisy signals and featureless spectra. We have developed numerical codes similar to those of Morente and coworkers, and we are able to duplicate the same spectra, supposedly observed during the descent. We then apply the same processes to the checkout data collected during the interplanetary transit, when the electric antenna is still stowed under the thermal shield of the Huygens Probe. The fact that similar spectral features are observed in both environments, namely, the atmosphere of Titan during the descent on the one hand and the spacecraft enclosure during the transit on the other hand, casts a serious doubt on the methodology of the analysis; it definitely rules out, if need be, the alleged evidence of several harmonics of the global ELF Schumann resonance and fundamental and harmonics of the VLF transverse resonance.

\section{Deciphering the Artifact}

[11] The flaw of the analysis performed by Morente et al. [2008] in their first ELF paper will be briefly apprehended in a simple way; the interested reader is referred to the work of Berthelin et al. [2009]. The waveform of the original signal is analyzed on board within a preset bandwidth and with a given resolution, and the outcome that consists of a number of evenly distributed spectral lines is transmitted to ground. Following the steps of Morente et al. [2008], assuming that all phases are equal to zero and ignoring calibration, an inverse DFT yields a pseudowaveform. A second DFT is finally applied once the temporal window has been both truncated (late time signal) and extended with a large number of zeroes, with the hope of improving the frequency resolution and enhancing features not visible in the raw spectrum. The end product [Morente et al., 2008, Figure 5] is a spectrum with an improved resolution that displays an impressive number of ideally spaced pseudoresonances.

[12] The truth is more prosaic and can be readily understood from a mathematical point of view. Let us note that framing a temporal window between strings of zeroes is equivalent to multiplying a waveform by a rectangular function. The transform of the product of two functions is the convolution of their transforms, namely (1) the sinc function and (2) the low-resolution spectrum that would be obtained without the adjunction of zeroes. The shape of the so-called resonances is more or less controlled by the width of the central peak of the sinc function.

[13] Let us point out, for further reference, that delaying the start of the late time signal by blanking out the first samples introduces a phase shift on the spectral components, proportional to the time delay, that increases linearly with frequency. This phase shift does not affect the DFT modulus but clearly influences the frequency distributions of its real and imaginary parts. This point is not relevant for the ELF signal analysis [Morente et al., 2008], but it is of paramount importance for understanding the treatment of the VLF signal [Morente et al., 2009b]. Since the VLF energy modulus distribution does not display sufficiently visible resonances, it seems that they show instead, without any explanation, the absolute value of the real part of the DFT. The effect derived from this operation is illustrated in section 4.

\section{Numerical Results}

[14] We have developed numerical tools that follow the steps and guidelines given in sections 2 and 3 . We first validate our own approach by duplicating the results already published by Morente and his colleagues. We use the same ELF and VLF data sets and apply our own specific treatments to the same late time signals, after adding an adequate quantity of zeroes to increase the total number of samples up to 960 and 320, respectively.

[15] Figure 1 shows the results obtained for the VLF signal. Contrary to the ELF spectrum, the VLF energy distribution (Figure 1, left) does not exhibit any remarkable feature, but plotting the absolute value of the real part of the DFT (Figure 1, right) seems to present more "attractive" characteristics. The real part of the energy distribution has no physical significance on its own and its only merit is to provide a fake spectrum offering a series of apparent resonances separated by intervals that are, this time, controlled by the phase shift induced by the early time blanking out [Berthelin et al., 2009]. Note the perfect similarity between Figure 1 (right) and the first spectrum in Figure 10d of Morente et al. [2009b].

[16] The application of our own codes to the ELF and VLF data recorded at an altitude of $141 \mathrm{~km}$ yields the results shown in Figures $2 \mathrm{a}$ and $2 \mathrm{~b}$ and $2 \mathrm{c}$ and $2 \mathrm{~d}$, respectively. The 

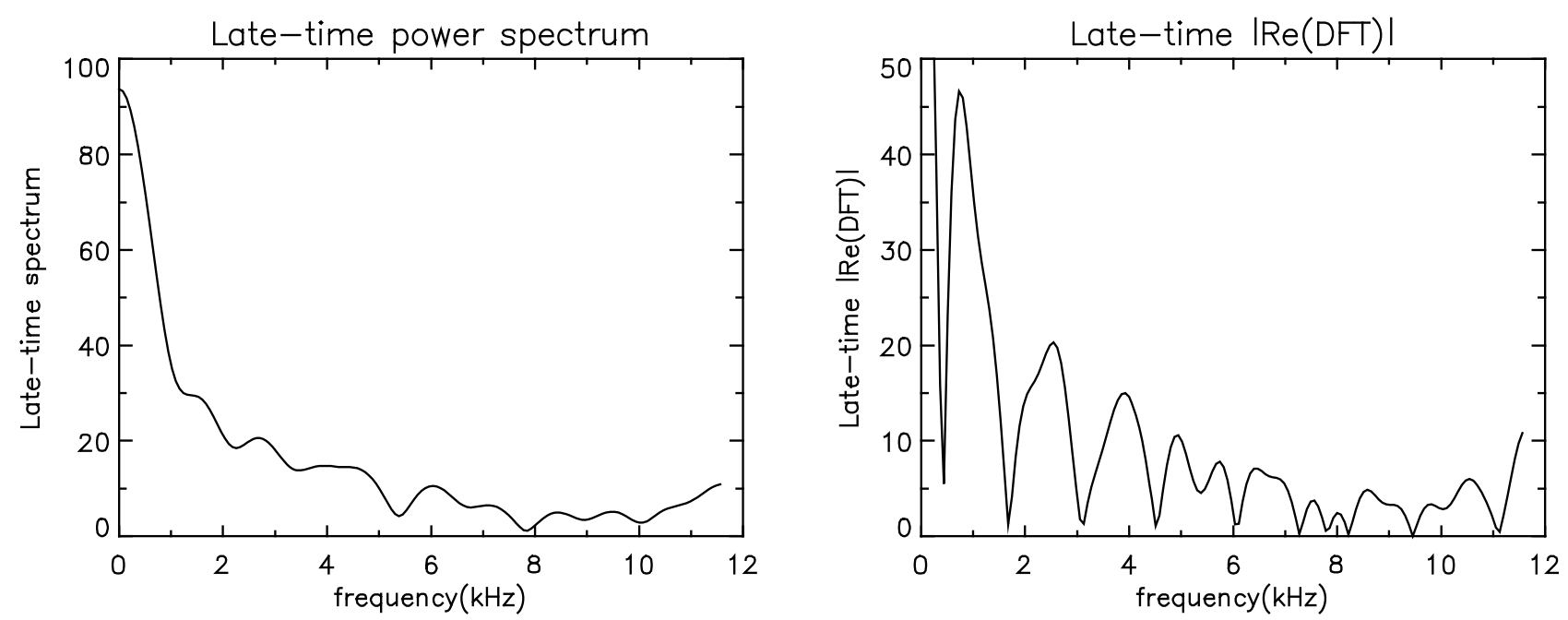

Figure 1. A comparison between (left) the modulus and (right) the absolute value of the real part of the spectral distribution derived with our code from the first VLF raw spectrum recorded at an altitude of $141 \mathrm{~km}$ (see text for detail).
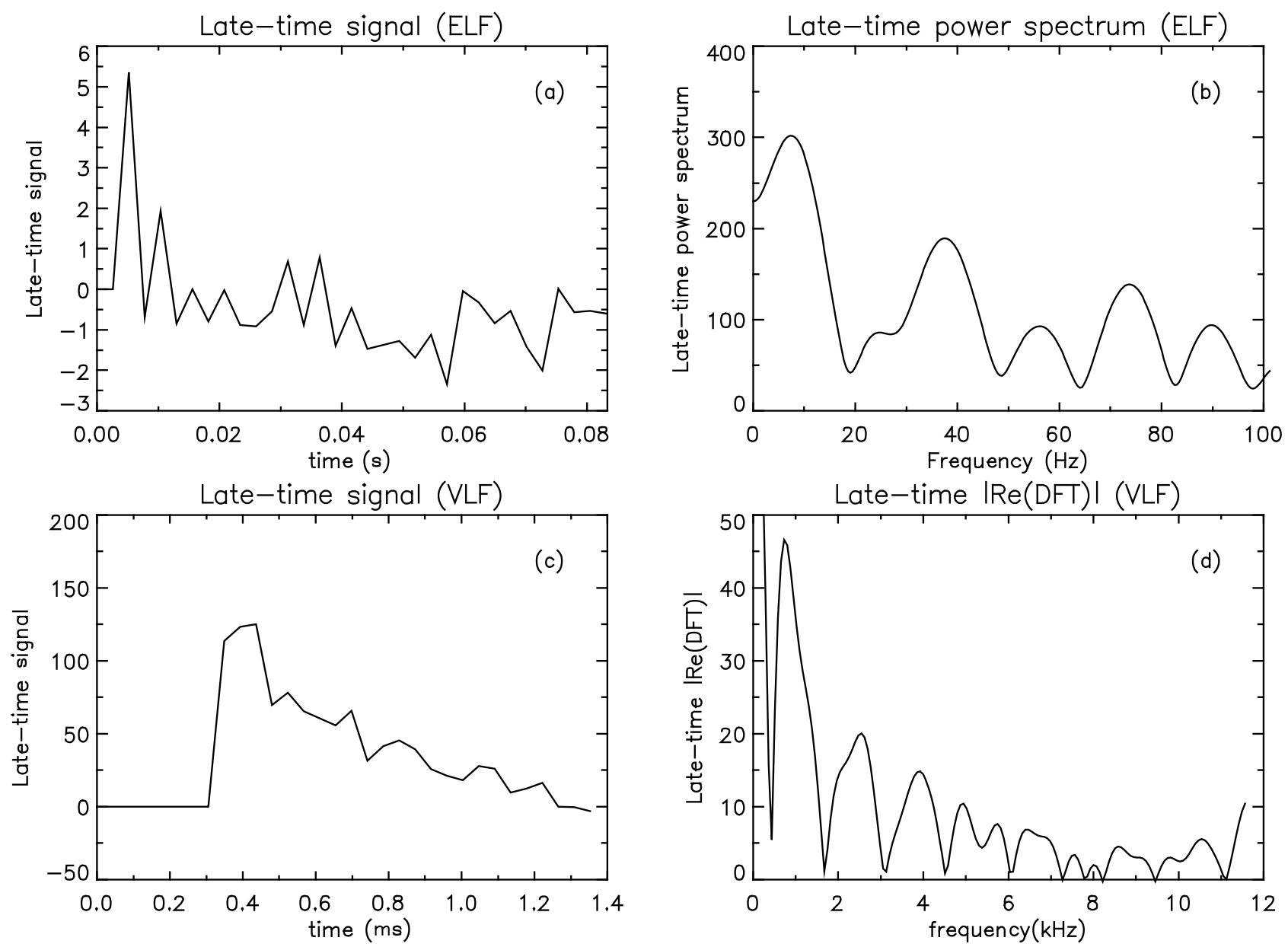

Figure 2. Results derived from the application of our codes to the ELF (top) and VLF (bottom) sequences recorded at an altitude of $141 \mathrm{~km}$. (a and c) The late time signals and (b and d) their associated frequency distributions are shown (Figure 2d same as Figure 1, right). 

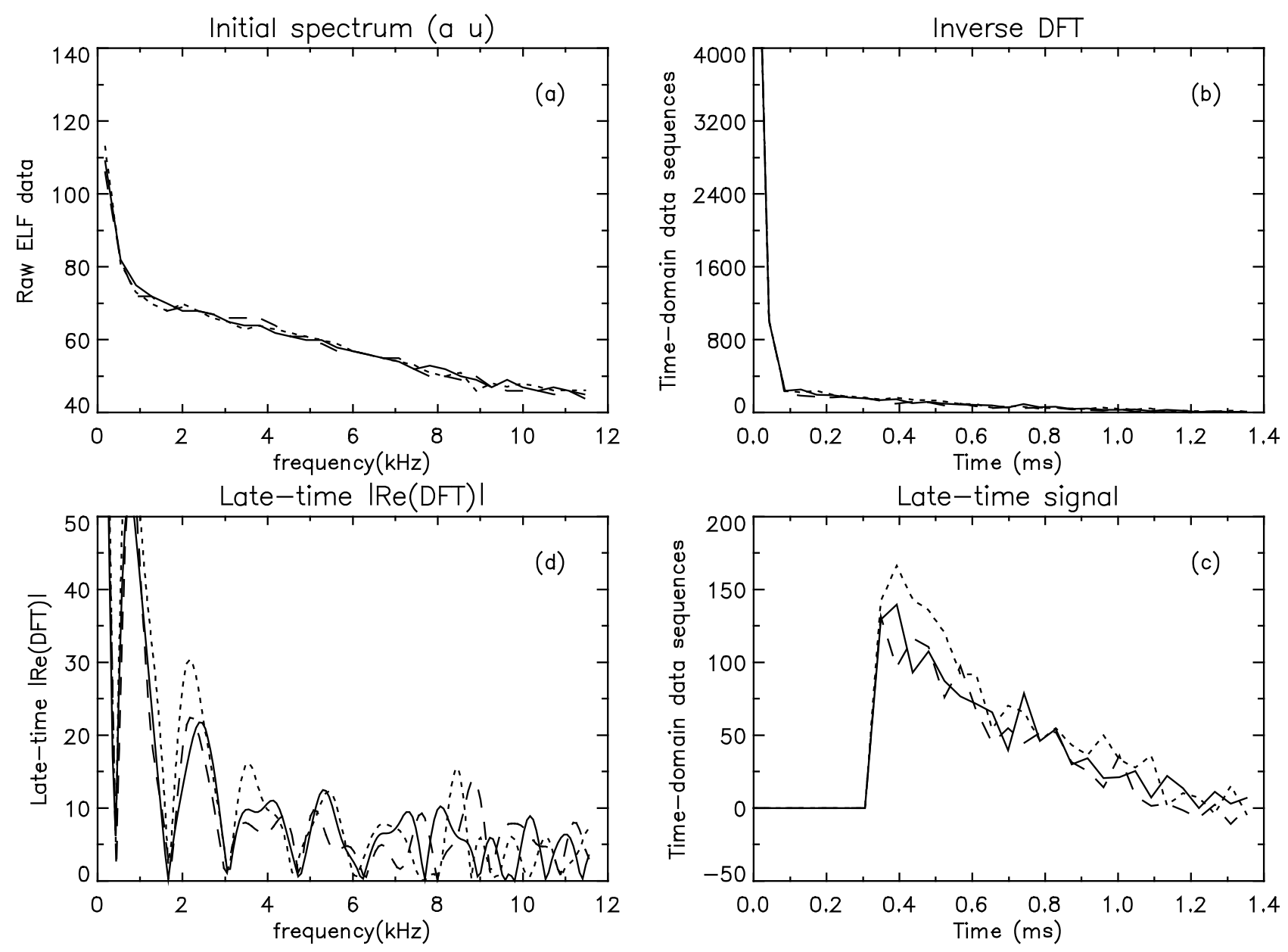

Figure 3. Application of our code to three VLF data sequences (solid, dot and dash lines) recorded during checkout session 10. The presentation is identical to that of Figure 10 of Morente et al. [2009b]. (a) Raw spectra. (b) Full-scale temporal late time function. (c) Same as Figure 3b with expanded amplitude and 8 zeros added in place of the early time response. (d) Absolute value of DFT's real part.

spectra shown on the right side are derived from the late time signals shown on the left side; they display peaks that have been asserted by Morente et al. to be associated with the Schumann and transverse resonances, and their harmonics. Figures $2 \mathrm{a}$ and $2 \mathrm{~b}$ should be compared with Figures $5 \mathrm{c}$ and 5d of Morente et al. [2008], and Figures 2c and 2d should be compared with Figures $10 \mathrm{c}$ and $10 \mathrm{~d}$ of Morente et al. [2009b]. The perfect match between our results and those of Morente and coworkers demonstrates the similarity of the two numerical approaches.

[17] We shall now apply our procedure to the data collected during a checkout session, before the release of the Huygens Probe from the Cassini Orbiter. The various steps of the treatment applied to successive VLF check-out sequences are given as examples in Figure 3 that shows the original raw spectra in arbitrary units (Figure 3a), the time series derived from the inverse DFT (Figure 3b), the late time signals obtained after blanking out the first samples (Figure 3c), and the final results that are all very similar to those shown in Figure 2d (Figure 3d). Comparing our Figure 3 and Figure 10 of Morente et al. [2009b] is very informative. The normalization factors of the signal ampli- tudes are different but Figures 2 and 3 are very similar, in spite of the fact that one deals with checkout data and the other one with flight data. Features observed during the interplanetary transit with an instrument that is not in a flight configuration cannot, beyond doubt, be the signatures of VLF electromagnetic resonances in the atmosphere of Titan. The same remark can be made about the ELF signal by comparing the checkout and flight data [Béghin et al., 2009].

\section{Conclusion}

[18] Since the two papers of Morente and coworkers rely on similar faulty approaches, the conclusion of our comment about their second VLF paper does not markedly differ from that about their first ELF paper [Hamelin et al., 2009]. We therefore refute the work of Morente and coworkers on the following grounds:

[19] 1. Their approach is suspicious from the beginning because they do not apprehend correctly the raw data that are neither calibrated, nor decompressed, nor even plotted in physical units. 
[20] 2. Due to the fact that the phases are arbitrarily assumed to be zero, the inverse DFT analysis necessarily yields an arbitrary signal with a meaningless peak at time zero; it cannot display any spectral component whose amplitude is less than that of the less significant bit in the original data set.

[21] 3. Adding zeroes before and/or after a time series, should be done with caution, especially if one knows nothing about the waveform. First, this operation does not contribute any new information contrary to increasing the sampling frequency. Second, the derived ELF and VLF spectral distributions are distorted and exhibit peaks at intervals that are essentially controlled by the duration of the late time signal or that of the initial blanking out period, respectively.

[22] 4. The modulus of the real part of the spectral distribution, in the case of the VLF signal analysis, has no physical significance.

[23] 5. The alleged ELF and VLF resonances are artifacts necessarily generated by the analysis procedure, since the spectral features observed during the interplanetary transit and during the descent are similar.

[24] Any progress in the understanding of the electrical state of Titan's atmosphere is of the highest significance to many scientists in numerous disciplines. It is therefore extremely important to firmly contradict all publications that contain erroneous information on this subject.

\section{Appendix A: Response to the Reply of Morente et al.}

\section{A1. Introduction}

[25] The main text consists of a critical evaluation of the work of Morente et al. [2009b] on the VLF electric field measurements performed with the PWA instrument during the descent of the Huygens Probe through the atmosphere of Titan. From Morente et al.'s reply it is likely that Morente and his coworkers did not understand completely our criticisms, and we therefore respond to their reply in this appendix, written after the main text. To avoid any confusion, we did not modify the original commentary, but we found it nevertheless necessary, for clarity, to expand our comments with this appendix, which includes additional remarks and develops further some of our earlier arguments.

\section{A2. Calibration and Decompression of the Raw Data}

[26] We first noted that Morente and his coworkers performed a spectral analysis on the raw telemetry data without calibration or decompression. The calibration corrects for the lack of linearity of the electronic circuitry and the purpose the logarithmic compression is to enlarge the dynamic range of the measurements [Hofe, 2005]. The major features of the original spectrum might still be visible in the raw data, but they are always grossly distorted [see Morente et al., 2009a, Figure 1a]. Disregarding that the instrument is not perfectly linear and that its output has been logarithmically compressed is an expeditious practice that displays a lack of rigor. Such an approach is never commendable and applying an inverse DFT to the raw data set, that is the logarithm of a spectrum ( $\mathrm{dB}$ scale), violates the basic rule that the linearity of the signal should not be distorted.

[27] Morente et al. [2009a] first argued that they used the raw data because the calibrated data were not available to them. This argument is not admissible since the raw data and the calibration document were released together in the ESA archive (and NASA mirror): ftp://psa.esac.esa. int/pub/mirror/CASSINI-HUYGENS/HASI/HP-SSAHASI-2-3-4-MISSION-V1.1/CALIB/PWA/HASI_PWA_ CALIBRATION_REPORT.PDF (28-6-2006).

[28] Their second explanation [Morente et al., 2011, equations (1)-(3)] is also unacceptable. They write "there is a linear relation between the raw data and the power spectrum." They should have written instead "there is a linear relation between the raw data and the logarithm of the power spectrum."

\section{A3. Significance of the Real Part of the Spectrum}

[29] We pointed out in the main text a consequence of the procedure developed by Morente et al. [2009b] that is illustrated in their Figure 10. They claimed and continue assuming in their reply that the absolute value of the real part of the DFT is representative of the power spectrum of the late time signal. We shall now prove that this allegation is totally unjustified from a physical point of view.

[30] Let us first recall that applying a Fourier transform (and DFT) to a real temporal signal generally yields a complex output. Only the modulus of the power density spectrum has a physical meaning and is defined by

$$
|\mathrm{DFT}|=\left(\mathrm{Re}_{\mathrm{DFT}}^{2}+\operatorname{Im}_{\mathrm{DFT}}^{2}\right)^{1 / 2},
$$

where

$$
\begin{gathered}
\mathrm{Re}_{\mathrm{DFT}}=|\mathrm{DFT}| \cos \varphi, \\
\mathrm{Im}_{\mathrm{DFT}}=|\mathrm{DFT}| \sin \varphi, \\
\varphi=\operatorname{atan} \frac{\mathrm{Im}_{\mathrm{DFT}}}{\mathrm{Re}_{\mathrm{DFT}}} .
\end{gathered}
$$

Since no phase information can be associated with any averaged energy spectrum, these authors assume $\varphi=0$ in equation (A2) at all frequencies and conclude that the modulus (equation (A1)) equals the absolute value of the real part (equation (A2)).

[31] This is incorrect since there is no reason that the DFT phases of the artificial late time signal built up by Morente et al. be zero at all frequencies, as demonstrated by Berthelin et al. [2009]. This condition can only be met if one also includes the symmetrical part of the late time response with respect to $t=0$, in other words, if one violates the causality principle. The modulus of the real part of the energy distribution has therefore no physical significance.

[32] The "late time signal" corresponding to the case study selected by Morente et al. [2009b] is shown in their Figure 10c and they claim that their Figure 10d represents its energy spectrum. The result of the DFT should in fact resemble the power spectrum shown in Figure 1 (left) and not replicate the spectral distribution shown in Figure 1 (right). Irrespective of the causality principle, this point is further developed in the following alternative explanation where we demonstrate that the artifact can easily be controlled by means of an adjustable parameter, the duration of the "early time" gap. 

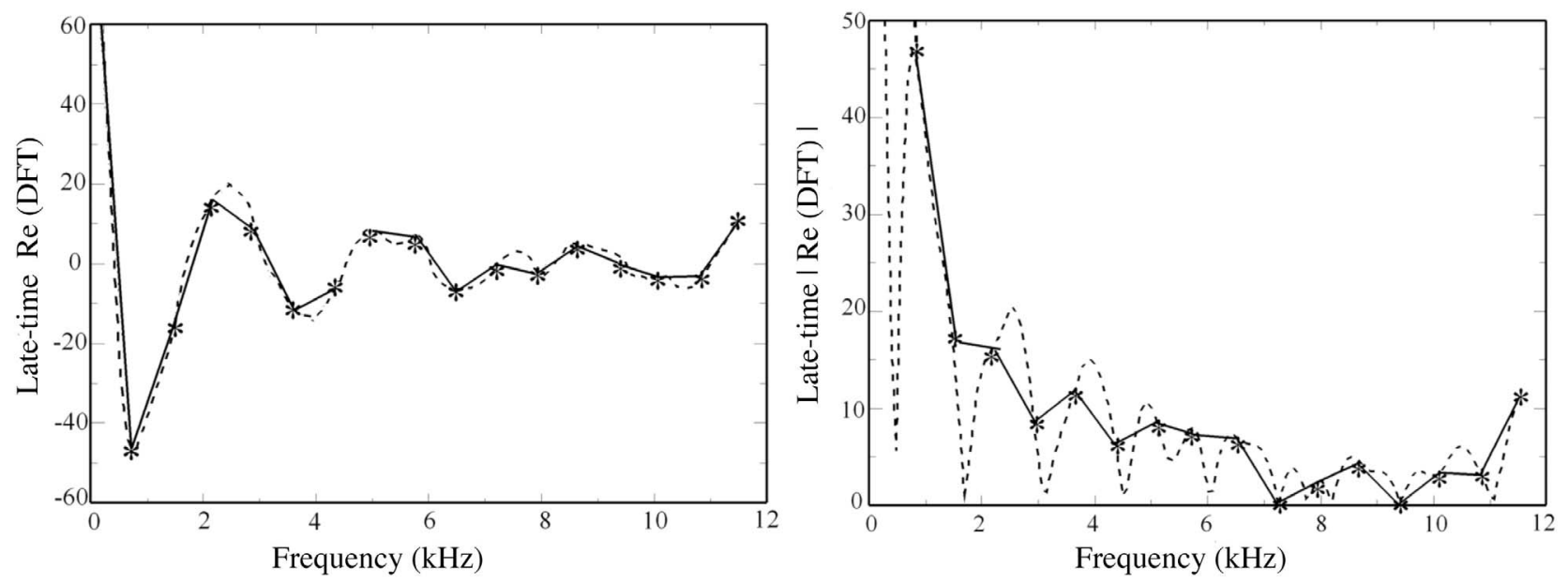

Figure A1. Late time response derived from the VLF sequence recorded at $141 \mathrm{~km}$ when the 8 early time samples are set to zero. (left) Algebraic value of the real part of the DFT. (right) absolute value of this real part. The asterisks and solid line interpolation indicate no zero added. The dashed lines indicate 288 zeros added. The dashed line in Figure A1 (right) is identical to the solid lines in Figure 10d of Morente et al. [2009b] and Figures 1 (right) and 2d.

[33] It is first seen in Figure A1 that the periodicity of the claimed resonances is related to the phase of the complex DFT vector that increases with frequency (for details, see Berthelin et al. [2009]). Assuming an "early time" gap of eight samples, the modulus and the algebraic value of the real part of the distribution are represented in Figures A1 (left) and A1 (right) with and without additional zeros, respectively (dashes and asterisks). The oscillations of the phase are visible in the two curves in Figure A1 (left). Extending the time series with zeros and rectifying the real part of the spectrum nicely reveals in Figure A1 (right) the pseudo resonances that are spaced at half the intervals that separate adjacent maxima in Figure A1 (left). The fact that due to the zero crossings of the real part, the ampli- tude vanishes completely between two maxima is not a usual characteristic of a real energy spectrum. This simple anomaly should have called the attention of Morente and his colleagues.

[34] Figure A2 shows the polar plots of the DFT vector with different "early time" gaps, 8 and 4 samples, respectively, but with the same number of added zeros. It is seen that the interval between adjacent pseudoresonances can be adjusted at will by modifying the duration of the blanking out period. This observation is not completely unrelated with the shift theorem, which states that shifting the starting time of a signal induces a phase rotation of its complex spectrum that increases with frequency and is proportional to the shift. As illustrated in Figure A2, the phase rotation at

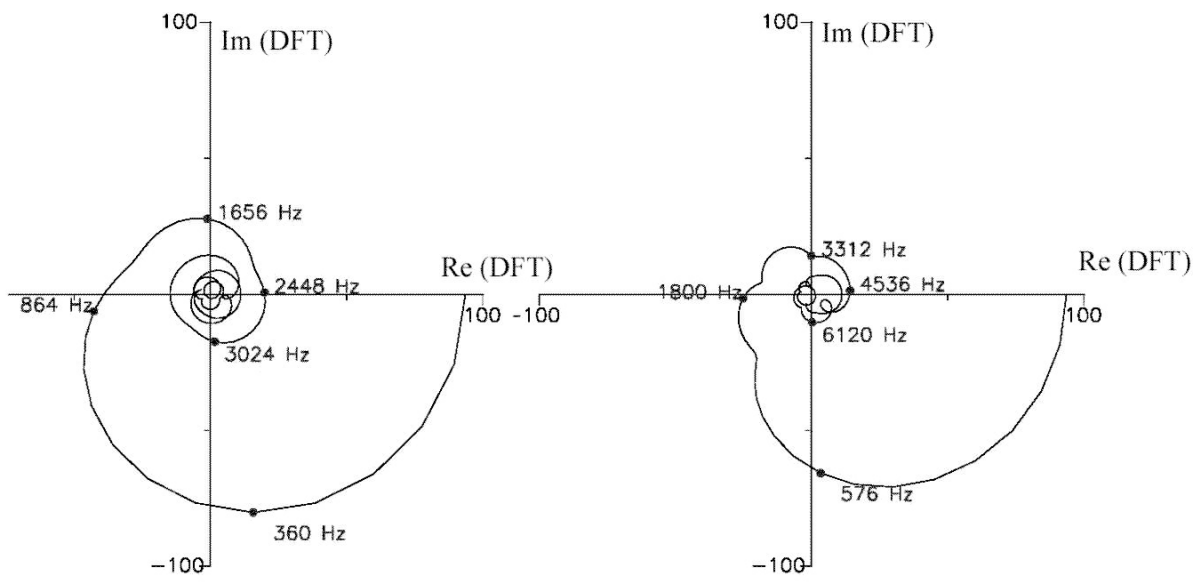

Figure A2. Polar representations of the complex DFT vector with 288 zeros added for two durations of the early time gap at (left) $0.347 \mathrm{~ms}$ (8 samples) and (right) $0.17 \mathrm{~ms}$ (4 samples). Figure A2 (left) and the dashed lines of Figure A1 are closely related. Comparing the two diagrams confirms that the intervals between successive pseudoresonances, i.e., maxima of $\mid \operatorname{Re}$ (DFT)|, are controlled by the duration of the gap. 
a given frequency is effectively larger when the duration of the "early time" gap is doubled. A gap of 8 samples gives an average separation between successive harmonics of $0.67 \mathrm{kHz}$ that supposedly fits very well the TLM prediction model of Morente et al. [2009b]. This claim is unfounded since we have proven that a gap of four samples would have approximately doubled this interval.

\section{A4. In-Flight Checkout Data}

[35] Morente et al. acknowledge that Berthelin et al. [2009] have successfully reconstructed their procedure and correctly duplicated the numerical results published in their paper.

[36] Since the application of the very same technique to the checkout data collected during the cruise yields spectra similar to those obtained with the descent data, we have irrefutably demonstrated that the pseudoresonances have no physical significance. These spectral features are necessarily artifacts of the analytical procedure developed by Morente and his team.

[37] They assert first that their numerical approach does not reveal any resonance where there is none, using a "timedamped" artificial signal exclusively made of exponential functions [see Morente et al., 2011, Figure 1]. The "amplitude spectrum" plotted in their Figure 1d is indeed featureless. Why do not they plot instead the absolute value of the real part of the spectral distribution, which would probably reveal nice oscillations similar to those obtained with the descent data?

[38] They then apply a similar treatment to a white noise sequence [Morente et al., 2011, Figure 2] and produce an amplitude spectrum that, they claim, is also white. After a closer examination of their Figure $2 \mathrm{~d}$, one could declare alternatively that this spectral distribution resembles a series of pseudoresonances. The facts that the minima of the amplitude are nearly equal to zero and that the peaks are spaced at regular intervals are suspicious.

[39] Finally, they perform a statistical study on the peak interval count distribution pertaining to the in-flight checkout session [Morente et al., 2011, Figure 3] and observe that it "shows a relatively flat shape." This assertion is questionable because both the amplitude and the frequency of the maximum count are quite similar to those observed during the descent [Morente et al., 2009b, Figure 11]. The apparent flatness of the count distribution is simply due to the fact that the scale along the ordinate axis is 16 times larger in Figure 3 of Morente et al. [2011] than in Figure 11 of Morente et al. [2009b].

\section{A5. Conclusion}

[40] The analysis and results by Morente et al. [2009b] are incorrect for several reasons. They assert that they can extract information from a signal without any consideration for calibration, data compression and signal-to-noise ratio. They ignore that it is impossible to retrieve a meaningful waveform from the compilations of several averaged power spectra that, by definition, carry no information about the phase. It is correct that one can restore at will the spectrum of a sample series by adding an unlimited number of zeros, but it must be kept in mind that (1) this approach cannot improve the frequency resolution of the original signal and
(2) the spectrum characterizes less the properties of the original signal than those of the sample series itself (especially if it has been significantly manipulated).

[41] More importantly, they ignore that their numerical approaches give similar results when they are applied to the ELF or VLF data collected during the in-flight checkout session and during the descent in the atmosphere of Titan. Finally, they make our VLF measurements fit their theoretical predictions, by resorting to the most unlikely assumptions: (1) they claim that the rectified value of the real part of the DFT yields the power spectrum, (2) they add a large number of zeros to their "late time signal" to disclose the pseudoresonances, and (3) they adjust the frequencies of these artifacts to fit their predictions by selecting the proper duration of the "early time" gap.

[42] Acknowledgment. The first author is indebted to Harri Laakso for valuable support and useful comments.

\section{References}

Béghin, C., et al. (2007), A Schumann-like resonance on Titan driven by Saturn's magnetosphere possibly revealed by the Huygens Probe, Icarus, 191, 251-266, doi:10.1016/j.icarus.2007.04.005.

Béghin, C., et al. (2009), New insights on Titan's plasma driven Schumann resonance inferred from Huygens and Cassini data, Planet. Space Sci., 57, 1872-1888, doi:10.1016/j.pss.2009.04.006.

Berthelin, S., C. Béghin, and D. Lagoutte (2009), Critical assessment of the late-time method used by Morente et al. (2008, 2009), PWA report, LPC2E, CNRS, Univ. of Orléans, Orléans, France. (Available at ftp://lpc2e.cnrs-orleans.fr/users/Christian_BEGHIN/pub/Titan papers/CRITICAL ANALYSIS sept2009.pdf.)

Falkner, P. (2004), Permittivitäts Wellen und Altimeter Analysator für das ESA/NASA Projekt Cassini-Huygens, Ph.D. thesis, Tech. Univ. of Graz, Graz, Austria.

Fulchignoni, M., et al. (2002), The characterization of Titan's atmosphere physical parameters by the Huygens Atmospheric Structure Instrument (HASI), Space Sci. Rev., 104, 395-431, doi:10.1023/A:1023688607077.

Grard, R., H. Svedhem, V. Brown, P. Falkner, and M. Hamelin (1995), An experimental investigation of atmospheric electricity and lightning activity to be performed during the descent of the Huygens Probe on Titan, J. Atmos. Terr. Phys., 57, 575-585, doi:10.1016/0021-9169(94)00082-Y.

Grard, R., et al. (2006), Electric properties and related physical characteristics of the atmosphere and surface of Titan, Planet. Space Sci., 54, 1124-1136, doi:10.1016/j.pss.2006.05.036.

Hamelin, M., R. Grard, J. J. López-Moreno, K. Schwingenschuh, C. Béghin, J. J. Berthelier, and F. Simões (2009), Comment on "Evidence of electrical activity on Titan drawn from the Schumann resonances sent by Huygens probe", by J. A. Morente, J. A. Portí, A. Salinas, E. A. Navarro [doi:10.1016/j.icarus.2008.02.004], Icarus, 204, 349-351, doi:10.1016/j. icarus.2009.01.031.

Hofe, R. (2005), Signal analysis of the electric and acoustic field measurements by the Huygens instrument HASI/PWA, Diploma thesis, Inst. of Broadband Commun., Univ. of Technol., Graz, Austria.

Morente, J. A., J. A. Portí, A. Salinas, and E. A. Navarro (2008), Evidence of electrical activity on Titan from the Schumann resonances sent by Huygens probe, Icarus, 195, 802-811, doi:10.1016/j.icarus.2008.02.004.

Morente, J., J. Porti, A. Salinas, and E. Navarro (2009a), Rebuttal to "Comments on "Evidence of electrical activity on Titan drawn from the Schumann resonance sent by Huygens probe" by J. A. Morente, J. A. Porti, A. Salinas, and E. A. Navarro (2009a), [2008, Icarus, doi:10.1016/j.icarus.2008.02.004], Icarus, 204, 352-354, doi:10.1016/ j.icarus.2009.05.006.

Morente, J. A., J. A. Portí, C. Blanchard, E. A. Navarro, and A. Salinas $(2009 \mathrm{~b})$, An analysis of VLF electric field spectra measured in Titan's atmosphere by the Huygens probe, J. Geophys. Res., 114, E06002, doi:10.1029/2008JE003324.

Morente, J. A., J. A. Porti, E. A. Navarro, and A. Salinas (2011), Reply to comment by R. Grard et al. on "An analysis of VLF electric field spectra measured in Titan's atmosphere by the Huygens probe," J. Geophys. Res., 116, E05006, doi:10.1029/2010JE003581.

Simões, F., et al. (2007), A new numerical model for the simulation of ELF wave propagation and the computation of eigenmodes in the atmosphere 
of Titan: Did Huygens observe any Schumann resonance? Planet. Space Sci., 55, 1978-1989, doi:10.1016/j.pss.2007.04.016.

C. Béghin and S. Berthelin, LPC2E-CNRS-Université d'Orléans, 3A, Av. Recherche Scientifique, F-45071 Orléans CEDEX 2, France.

J.-J. Berthelier and M. Hamelin, LATMOS-IPSL-Université de Versailles St Quentin, 4, Av. de Neptune, F-94107 Saint-Maur, France.
R. Grard, Prinses Margrietlaan 4, NL-2341 VJ Oegstgeest, Netherland. (rgrard@rssd.esa.int)

J. J. López-Moreno, Instituto de Astrofisíca de Andalucía IAA-CSIC, Camino Bajo de Huetor 50, E-18008 Granada, Spain.

F. Simões, NASA Goddard Space Flight Center, 8800 Greenbelt Rd. Greenbelt, MD 20771, USA. 